\title{
ON THE NUMBER OF COMPONENTS OF A GRAPH RELATED TO CHARACTER DEGREES
}

\author{
O. MANZ, R. STASZEWSKI AND W. WILLEMS \\ (Communicated by Bhama Srinivasan)
}

\begin{abstract}
We connect two nonlinear irreducible character of a finite group $G$ if their degrees have a common prime divisor. In this paper we show that the corresponding graph has at most three connected components.
\end{abstract}

1. Introduction and the main result. Let $G$ be a finite group and let $\operatorname{Irr}(G)$ denote the set of the irreducible complex characters of $G$. We define a graph $\Gamma=\Gamma(G)$ in the following way.

The vertices of $\Gamma$ are represented by the nonlinear irreducible characters of $G$. We connect two vertices $\chi$ and $\varphi$ of $\operatorname{Irr}(G)$ if the degrees $\chi(1)$ and $\varphi(1)$ have a common prime divisor. Note here that $G$ is abelian if and only if $G$ is attached to the empty graph.

If $n(\Gamma)$ denotes the number of the connected components of $\Gamma$, we have the following main result.

THEOREM. $n(\Gamma(G)) \leq 3$ for any group $G$.

Unfortunately, the proof of the theorem relies on the classification of all finite simple groups. Let us here mention that for a solvable group we even have $n(\Gamma(G)) \leq 2$. This was proved by the first author in [9, Proposition 2]. Finally, these bounds are best possible; for instance, take $\operatorname{PSL}\left(2,2^{a}\right)(a \geq 2)$ in case of a nonsolvable, and the symmetric group $S_{4}$ in case of a solvable group.

As an application we obtain the following result which was proved by the first author in [8] under the hypothesis of a special case of Brauer's height zero conjecture and in full generality by the third author [17].

COROLlARY. Suppose that $G$ is a nonsolvable group and that the degrees of all irreducible complex characters of $G$ are powers of primes. Then $G \cong S \times A$ where $A$ is abelian and $S \in\left\{A_{5}, \mathrm{SL}(2,8)\right\}$.

PrOOF. By [8, Hilfssätze 3 und 5], we may assume that $G$ is simple. Furthermore, since the character degrees are powers of primes, a connected component $\Gamma_{i}$ of $\Gamma=\Gamma(G)$ contains only characters $\chi$ the degree of which is a power of a fixed prime $p_{i}$. By the theorem $n(\Gamma) \leq 3$, and by [8, Einleitung] we may even assume that $n(\Gamma)=3$. Now Michler's result $[\mathbf{1 1}, 5.4]$ implies that $G$ is a $\left\{p_{1}, p_{2}, p_{3}\right\}$-group and Satz 2 of $[8]$ yields $G \cong A_{5}$ or $G \cong \mathrm{SL}(2,8)$.

Received by the editors September 24, 1986 and, in revised form, March 1, 1987.

1980 Mathematics Subject Classification (1985 Revision). Primary 20C15.

The main part of this paper was written during the AMS meeting in Arcata 1986. The authors would like to thank the DFG and the 'Freunde der Universität Mainz' for their generous support. 
2. Reduction to simple groups. In order to prove the theorem, we reduce the problem to simple groups and check the degree configurations in the known list. Since many of the simple groups tend to have a connected graph, the following key proposition leads in most cases to a better bound than 3 .

Proposition. Suppose that $G$ is a nonsolvable group. Then

$$
n(\Gamma(G)) \leq \max _{E}\{n(\Gamma(E))\}
$$

where $E$ runs through the set of the simple nonabelian composition factors of $G$.

ProOF. Let $N$ be a maximal normal subgroup of $G$ and let

$$
k=\max _{E}\{n(\Gamma(E))\}
$$

where $E$ runs through the set of simple nonabelian composition factors of $G$. First we deal with the case that $G / N$ is simple and nonabelian. Clearly, there exist connected components $\Gamma_{1}, \ldots, \Gamma_{s}$ of $\Gamma=\Gamma(G)$ such that $s \leq k$ and $\{\varphi \mid \varphi \in \operatorname{Irr}(G), N \subseteq$ $\operatorname{Kern} \varphi, \varphi(1) \neq 1\} \subseteq \Gamma_{1} \cup \cdots \cup \Gamma_{s}$. Suppose $n(\Gamma)>k$ and choose a component of $\Gamma$, say $\Gamma_{k+1}$ which is different from $\Gamma_{i}(i=1, \ldots, s)$. Let $\chi \in \Gamma_{k+1}$. As $(\chi(1), \varphi(1))=1$ for all $\varphi \in \operatorname{Irr}(G / N)$, we obtain $(\chi(1),|G / N|)=1$ by Michler's Theorem 5.4 of $[\mathbf{1 1}]$. Thus $\chi_{N} \in \operatorname{Irr}(N)$ and Gallagher's theorem [6, 6.17] yields $\chi \varphi \in \operatorname{Irr}(G)$ for all $\varphi \in \operatorname{Irr}(G / N)$. However, $\chi \varphi \in \Gamma_{k+1}$, which forces $\varphi \in \Gamma_{k+1}$ for all $\varphi$ with $\varphi(1) \neq 1$ contrary to the choice of $\Gamma_{k+1}$. Thus it remains to deal with the case that $G / N$ is of prime order $p$. By the inductive hypothesis we have $n(\Gamma(N)) \leq k$. Furthermore, if there exists $\chi \in \operatorname{Irr}(N)$ with $p \mid \chi(1)$, then we are done. Thus we may assume that $p$ does not divide the degree of any irreducible character of $N$. Applying $[\mathbf{1 1}, 5.4]$ again we obtain that $N$ has a normal abelian Sylow $p$-subgroup $P$. Now suppose that there exists a nonlinear character $\psi \in \operatorname{Irr}(N)$ with inertial group $I_{G}(\psi)=N$. Then $\psi^{G} \in \operatorname{Irr}(G)$ with $p \mid \psi^{G}(1)$ and therefore $n(\Gamma(G)) \leq n(\Gamma(N)) \leq k$. Thus we are left with the case that in particular all nonlinear irreducible characters of $N / P$ are fixed by $G / P$. Suppose $P_{0}$ is a Sylow $p$-subgroup of $G$ and $\left[P_{0} / P, N / P\right] \neq 1$. Then a result of Isaacs ([7]; cf. [10]) implies that $N / P$ is solvable, a contradiction to the hypothesis of the Proposition. Thus we may assume that $P_{0} / P$ acts trivially on $N / P$. Therefore $P_{0} \unlhd G$. Now start the procedure again with $N_{0}$ maximal normal in $G$ and $P_{0} \leq N_{0}$. If $G / N_{0}$ is nonabelian, the same argument as above works. In case $\left|G / N_{0}\right|=q$ is prime, we obtain again that a Sylow $q$-subgroup $Q_{0}$ of $G$ is normal. Continuing this way, we finally end up with a solvable group $G$, which contradicts the hypothesis of the Proposition.

3. Sporadic and alternating groups. To complete the proof of the Theorem we have to calculate $n(\Gamma(G))$ for all finite nonabelian simple groups.

By checking the character degrees of the 26 sporadic groups given in [3], we obtain

PROPOSITION. If $G$ is one of the sporadic simple groups, then $\Gamma(G)$ is connected.

For the rest of this section, let $G$ be one of the simple alternating groups $A_{n}$. If $G \cong A_{5} \cong \mathrm{SL}(2,4)$, the character degrees are $1,3,4$, and 5, so that $n\left(\Gamma\left(A_{5}\right)\right)=3$. 
For $G \cong A_{6} \cong \operatorname{PSL}(2.9)$, the degrees are $1,5,8,9$ and 10 , hence $n\left(\Gamma\left(A_{6}\right)\right)=2$. Finally, if $G \cong A_{8}$, the graph is connected since we have character degrees 1,7 , $20,21,28,35,45,56,64$ and 70 . All the other alternating simple groups have a connected graph. We even obtain

PROPOSITION. If $n=7$ or $n \geq 9$, then for any odd prime $p \leq n$, the alternating group $A_{n}$ has an irreducible character $\chi$ such that $2 p \mid \chi(1)$.

PROOF. It is sufficient to show that there either exists an irreducible character $\chi$ of $S_{n}$ which is not selfadjoint such that $2 p \mid \chi(1)$ or there exists an irreducible character $\chi$ of $S_{n}$ such that $4 p \mid \chi(1)$.

The irreducible characters of $S_{n}$ are indexed by partitions and we consider the partition $\left(n-s-r, s+1,1^{r-1}\right)$, which corresponds to the following Young tableau.

$$
r\left\{\begin{array}{ccrc}
n-s & n-s-r & n-s-r-1 \cdots n-2 s-r+1 & n-2 s-r-1 \cdots 1 \\
r+s & s & s-1 & \cdots
\end{array}\right.
$$

Such a partition of course exists, if $r \geq 1, s \geq 0$ and $r+2 s+1 \leq n$. Furthermore. the diagram is selfadjoint exactly in the following cases

(i) $s=0$ and $n=2 r+1$,

(ii) $s=1$ and $n=2 r+2$.

Now, if $\chi \in \operatorname{Irr}\left(S_{n}\right)$ is the character corresponding to the partition $(n-s-r$, $\left.s+1,1^{r-1}\right)$, we have

$$
\begin{aligned}
\chi(1) & =\frac{n !(n-2 s-r)}{s !(r-1) !(n-s-r) !(r+s)(n-s)} \\
& =\left(\begin{array}{c}
n \\
s
\end{array}\right)\left(\begin{array}{c}
n-s-1 \\
r-1
\end{array}\right) \frac{n-2 s-r}{r+s}
\end{aligned}
$$

Case 1. Let $n$ be odd.

If we take in this situation $r$ to be odd and $s$ to be even, then $2 \mid \chi(1)$.

(i) Furthermore, let $n \not \equiv 0,-1(\bmod p)$.

We put $r:=p-2$ and $s:=0$. Then $r+2 s+1=p-1 \leq n$ and the partition is well defined. This specification yields

$$
\chi(1)=\left(\begin{array}{l}
n-1 \\
p-3
\end{array}\right) \frac{n-(p-2)}{p-2}=\left(\begin{array}{l}
n-1 \\
p-2
\end{array}\right)
$$

and $p \mid \chi(1)$, as $n \not \equiv 0,-1(\bmod p)$. Now, if $n \neq 2 p-3, \chi$ is not selfadjoint and we are done. For $n=2 p-3$, we take $r:=3$ and $s:=p-5$. Then $r+2 s+1=2 p-6 \leq n$ and

$$
\chi(1)=\frac{(2 p-3) ! \cdot 4}{(p-5) ! \cdot 2 \cdot(p-1) !(p-2)(p+2)}
$$

hence $2 p \mid \chi(1)$. If $p \geq 7, \chi$ is not selfadjoint, and if $p=5$, we have $\chi(1)=20$. 
(ii) Let $n \equiv 0(\bmod p)$.

If $p \neq 3$, we choose $r:=1$ and $s:=2$. Then $r+2 s+1=6 \leq n$ and

$$
\chi(1)=\frac{n !(n-5)}{2 \cdot(n-3) ! \cdot 3 \cdot(n-2)},
$$

so that $2 p \mid \chi(1)$. Here $\chi$ is not selfadjoint. If $p=3$, we put $r:=3$ and $s:=2$. Then $r+2 s+1=8 \leq n$,

$$
\chi(1)=\frac{n !(n-7)}{2 \cdot 2 \cdot(n-5) ! \cdot 5 \cdot(n-2)},
$$

$2 p \mid \chi(1)$ and $\chi$ is not selfadjoint.

(iii) Let $n \equiv-1(\bmod p)$.

If $n \neq 2 p-1$ and $n \neq 4 p-1$, we choose $r:=2 p-1$ and $s:=0$. We obtain $r+2 s+1=2 p \leq n$ and

$$
\chi(1)=\left(\begin{array}{c}
n-1 \\
2 p-2
\end{array}\right) \frac{n+1-2 p}{2 p-1} .
$$

Thus $2 p \mid \chi(1)$ and $\chi$ is not selfadjoint, as $n \neq 4 p-1$. So suppose that $n=2 p-1$. For $p=5$, we get $n=9$ and we consider the partition $(5,2,2)$. The corresponding character has degree 120 . For $p \geq 7$, we take $r:=p$ and $s:=2$. Then $r+2 s+1=$. $p+5 \leq n$ and

$$
\chi(1)=\frac{(2 p-1) !(p-5)}{2 \cdot(p-1) !(p-3) !(p+2)(2 p-3)},
$$

which means $2 p \mid \chi(1)$. We now have to assume that $n=4 p-1$. If $p=3$, we choose $r:=1$ and $s:=4$. Then $r+2 s+1=10 \leq n$ and $\chi(1)=132$ is divisible by 6 . For $p \geq 5$, we again take $r:=p$ and $s:=2$. So, we have $r+2 s+1=p+5 \leq n$ and

$$
\chi(1)=\frac{(4 p-1) !(3 p-5)}{2 \cdot(p-1) !(3 p-3) !(p+2)(4 p-3)}
$$

from which we conclude that $2 p \mid \chi(1)$.

Case 2. Let $n$ be even.

If we take now $r$ to be even and $s$ to be odd, we also have $2 \mid \chi(1)$.

(i) Suppose, that $n \not \equiv 1,-2(\bmod p)$.

If $p \neq 3$, we take $r:=p-3$ and $s:=1$. Then $r+2 s+1=p \leq n$ and

$$
\chi(1)=\left(\begin{array}{l}
n-2 \\
p-4
\end{array}\right) \frac{n(n-p+1)}{p-2} .
$$

It follows $4 p \mid \chi(1)$, as $n \not \equiv 1,-2(\bmod p)$. For $p=3$, let $r:=6$ and $s:=1$. We obtain $r+2 s+1=9 \leq n$, because $n \neq 8$. Furthermore

$$
\chi(1)=\left(\begin{array}{c}
n-2 \\
5
\end{array}\right) \frac{n(n-8)}{7},
$$

hence $4 p \mid \chi(1)$.

(ii) We now assume that $n \equiv 1(\bmod p)$.

If $n \geq 3 p+1$, we take $r:=2$ and $s:=p$. Thus $r+2 s+1 \leq n$.

$$
\chi(1)=\left(\begin{array}{l}
n \\
p
\end{array}\right) \frac{(n-p-1)(n-2 p-2)}{p+2}
$$


now implies $2 p \mid \chi(1)$. Thus, let $n=p+1$. (Note that $n$ is even.) Then $p>7$ and for $r:=2, s:=3$, we obtain $r+2 s+1=9 \leq n$ and

$$
\chi(1)=\left(\begin{array}{c}
p+1 \\
3
\end{array}\right) \frac{(p-3)(p-7)}{5}
$$

which yields that $4 p \mid \chi(1)$.

(iii) Finally we have to consider $n \equiv-2(\bmod p)$. Furthermore, we may assume that $p>3$, because otherwise we are done due to (ii). If $n \geq 4 p-2$, we choose $r:=2 p$ and $s:=1$, so that $r+2 s+1=2 p+3 \leq n$. Since

$$
\chi(1)=\left(\begin{array}{c}
n-2 \\
2 p-1
\end{array}\right) \frac{n(n-2 p-2)}{2 p+1}
$$

we get $4 p \mid \chi(1)$. So let $n=2 p-2$. Surely $p \neq 5$, since $n \neq 8$. For $p=7$, we take $r:=1$ and $s:=2$ and obtain $\chi(1)=154$. If $p>7$, we choose $r:=p-1$ and $s:=3$. We get $r+2 s+1=p+6 \leq n$ and

$$
\chi(1)=\left(\begin{array}{c}
2 p-2 \\
3
\end{array}\right)\left(\begin{array}{c}
2 p-6 \\
p-2
\end{array}\right) \frac{p-7}{p+2} .
$$

Thus $2 p \mid \chi(1)$ and the proof is complete.

4. Simple groups of Lie type. In this section we freely use the following known facts (cf. [14 and 13, §11, §12]):

Let $L$ be a finite simple group of Lie type. Then there exists a simple, simply connected, linear algebraic group $G$ and an endomorphism $\sigma$ of $G$ such that (i) $G_{\sigma} / Z\left(G_{\sigma}\right) \cong L$ or (ii) $L$ is a proper subgroup of $L_{0} \cong G_{\sigma} / Z\left(G_{\sigma}\right)=G_{\sigma}$ which occurs exactly in the following cases:

\begin{tabular}{l|c|l|l}
$G_{\sigma}=L_{0}$ & $\left|L_{0}: L\right|$ & $L=L_{0}^{\prime}$ & \\
\hline$B_{2}(2)$ & 2 & $A_{6}=A_{1}(9)$ & \\
$G_{2}(2)$ & 2 & ${ }^{2} A_{2}(3)$ & \\
${ }^{2} F_{4}(2)$ & 2 & ${ }^{2} F_{4}(2)^{\prime}$ & Tits' simple group \\
${ }^{2} G_{2}(3)$ & 3 & $A_{1}(8)$ &
\end{tabular}

For some of the groups, the main result has to be checked separately.

4.1 LEMMA. $n(\Gamma(L)) \leq 3$ for each of the following simple groups:

$$
\begin{aligned}
& A_{1}(q)(q>3), A_{2}(q),{ }^{2} A_{2}(q)(q>2),{ }^{2} B_{2}\left(2^{2 m+1}\right)(m \geq 1), \\
& { }^{3} D_{4}(q), G_{2}(q)(q>2),{ }^{2} G_{2}\left(3^{2 m+1}\right)(m \geq 1),{ }^{2} F_{4}(2){ }^{\prime} .
\end{aligned}
$$

PROOF. This can almost be done checking the known character tables.

\begin{tabular}{ll} 
Group & Reference \\
\hline$A_{1}(q)$ & {$[\mathbf{1}$, Chapter XI, §5] } \\
$A_{2}(q)$ & {$[\mathbf{1 2}]$} \\
${ }^{2} A_{2}(q)$ & {$[\mathbf{1 2}]$} \\
${ }^{2} B_{2}\left(2^{2 m+1}\right)$ & {$[\mathbf{1}$, Chapter XI, §5], if $m>1$} \\
${ }^{3} D_{4}(q)$ & {$[\mathbf{4}]$} \\
$G_{2}(q)$ & {$[\mathbf{2}]$, if 2,3 does not divide $q$} \\
$G_{2}\left(3^{m}\right)$ & {$[\mathbf{5}]$} \\
${ }^{2} G_{2}\left(3^{2 m+1}\right)$ & {$[\mathbf{1 6}]$} \\
${ }^{2} F_{4}(2)^{\prime}$ & {$[\mathbf{3}]$}
\end{tabular}


Furthermore, the smallest Suzuki group ${ }^{2} B_{2}(8)$ has character degrees $1,2^{6}, 14$, $65,35,91$ (see [3]). Thus only the groups $G_{2}(q)$ in characteristic 2 are left. Let $L=G_{2}(q), q=2^{m}$ with $m>1$. By $[15, \S 17], L$ has two maximal anisotropic tori $G_{2}$ and $A_{2}$ of orders $q^{2}-q+1$ and $q^{2}+q+1$ respectively. Moreover, both tori contain regular elements, hence characters in general position since $G_{2}$ and $A_{2}$ are cyclic.

Thus we obtain two irreducible characters $\chi$ and $\psi$ of $L$ with degrees

$$
\chi(1)=\left|L: G_{2}\right|_{2^{\prime}}=(q-1)^{2}(q+1)^{2}\left(q^{2}+q+1\right)
$$

and

$$
\psi(1)=\left|L: A_{2}\right|_{2^{\prime}}=(q-1)^{2}(q+1)^{2}\left(q^{2}-q+1\right)
$$

(see $\left[11\right.$, Proposition 2.2]). Since $|L|=q^{6}(q-1)^{2}(q+1)^{2}\left(q^{2}+q+1\right)\left(q^{2}-q+1\right)$, the assertion follows.

4.2 LEMMA. Suppose that $L=G_{\sigma} / Z\left(G_{\sigma}\right)$ is a finite simple group of Lie type defined over the field $G F(q)$ where $q=r^{m}$ with $r$ a prime. Furthermore assume that the following two conditions hold.

(i) There exist two $\sigma$-stable maximal tori, say $T^{1}$ and $T^{2}$ of $G$ such that whenever $p$ is a prime divisor of $\left(\left|T_{\sigma}^{1} / Z\left(G_{\sigma}\right)\right|,\left|T_{\sigma}^{2} / Z\left(G_{\sigma}\right)\right|\right)$ then $p|| G_{\sigma} / Z\left(G_{\sigma}\right): T_{\sigma}^{i} / Z\left(G_{\sigma}\right) \mid$ for some $i \in\{1,2\}$.

(ii) There exist $\chi_{1}, \chi_{2} \in \operatorname{Irr}\left(G_{\sigma} / Z\left(G_{\sigma}\right)\right)$ with $\chi_{i}(1)=\left|G_{\sigma} / Z\left(G_{\sigma}\right): T_{\sigma}^{i} / Z\left(G_{\sigma}\right)\right|_{r^{\prime}}$. Then $n(\Gamma(L)) \leq 3$.

ProOF. The reader may check that $\Gamma(L)$ consists of the components corresponding to $\chi_{1}, \chi_{2}$ and the Steinberg character.

Now a careful reading of the proofs in Michler's paper [11] yields

4.3 LEMMA. All the assumptions of Lemma 4.2 can be fulfilled for any simple group $L$ of Lie type unless $L$ is isomorphic to one of the groups listed in 4.1.

This completes the proof of the Theorem.

ACKNOWLEDGEMENT. We would like to thank the referee for pointing out some very helpful comments.

ADDED IN PROOF. In a more natural way the following graph $\Gamma$ may be attached to the character degrees. The vertices of $\Gamma$ are represented by the elements of

$$
\rho(G)=\{p \mid p \text { a prime, } p \mid \chi(1) \text { for some } \chi \in \operatorname{Irr}(G)\} \text {. }
$$

Two different primes $p_{1}, p_{2}$ of $\rho(G)$ are connected if there exists a $\chi \in \operatorname{Irr}(G)$ such that $p_{1} p_{2} \mid \chi(1)$. Then the results of this paper still hold for this graph with essentially the same proofs.

\section{REFERENCES}

1. N. Blackburn and B. Huppert, Finite groups. III, Springer-Verlag, Berlin, 1982.

2. B. Chang and R. Ree, The characters of $G_{2}(q)$, Symposia Math. 13 (1974), 395-413.

3. J. H. Conway, R. T. Curtis, S. P. Norton, R. A. Parker and R. A. Wilson, Atlas of finite groups, Clarendon Press, Oxford, 1985.

4. D. I. Deriziotis and G. O. Michler, Character table and blocks of finite simple triality groups ${ }^{3} D_{4}(q)$, Trans. Amer. Math. Soc. 303 (1987), 39-70.

5. H. Enomoto, The characters of the finite Chevalley group $G_{2}(q), q=3^{f}$, Japan J. Math. 2 (1976), 191248. 
6. M. I. Isaacs, (haracter theory of finite groups, Academic Press, New York, 1976.

7. __ Coprime group actions fixing all nonlinear irreducible characters, Canad. J. Math. (submitted).

8. O. Manz, Endliche nicht-auflösbare Gruppen, deren sämtliche Charaktergrade Primzahlpotenzen sind, J. Algebra 96 (1985), 114-119.

9. __ Degree problems. II, П-separable character degrees, Comm. Algebra 13 (1985), 2421-2431.

10. O. Manz and R. Staszewski, Some applications of a fundamental theorem by Gluck and Wolf in the character theory of finite groups, Math. Z. 192 (1986), 383-389.

11. G. Michler, A finite simple group of Lie-type has $p$-blocks with different defects, $p \neq 2$, J. Algebra 104 (1986), 220-230.

12. W. A. Simpson and J. S. Frame, The character tables for $\operatorname{SL}(3, q), \operatorname{SU}\left(3, q^{2}\right), \operatorname{PSL}(3, q)$, $\operatorname{PSU}\left(3, q^{2}\right)$, Canad. J. Math. 25 (1979), 486-494.

13. R. Steinberg, Endomorphisms of linear algebraic groups, Mem. Amer. Math. Soc. No. 80, (1968), $108 \mathrm{pp}$.

14. J. Tits, Algebraic and abstract simple groups, Ann. of Math. (2) 80 (1964), 313-329.

15. F. D. Veldkamp, Regular elements in anisotropic tori, Contributions to Algebra, Academic Press, New York 1977, pp. 389-424.

16. H. N. Ward, On Ree's series of simple groups, Trans. Amer. Math. Soc. 121 (1966), 62-89.

17. W. Willems, Blocks of defect zero and degree problems, Proc. Sympos. Pure Math., vol. 47, Part I, Amer. Math. Soc., Providence, R. I., 1987, pp. 481-484.

Department of Mathematics, University of Mainz, 6500 Mainz, West GerMANY 